\title{
Entanglement of Formation of Bipartite Quantum States
}

\author{
Kai Chen $^{1}$, Sergio Albeverio ${ }^{1}$, and Shao-Ming Fei ${ }^{1,2,3}$ \\ ${ }^{1}$ Institut für Angewandte Mathematik, Universität Bonn, D-53115, Germany \\ ${ }^{2}$ Department of Mathematics, Capital Normal University, Beijing 100037, China \\ ${ }^{3}$ Max Planck Institute for Mathematics in the Sciences, D-04103 Leipzig, Germany
}

\begin{abstract}
We give an explicit tight lower bound for the entanglement of formation for arbitrary bipartite mixed states by using the convex hull construction of a certain function. This is achieved by revealing a novel connection among the entanglement of formation, the well-known Peres-Horodecki and realignment criteria. The bound gives a quite simple and efficiently computable way to evaluate quantitatively the degree of entanglement for any bipartite quantum state.
\end{abstract}

PACS numbers: 03.67.Mn, 03.65.Ud, 89.70.+c

Quantum entangled states are used as key resources in quantum information processing and communication, such as in quantum cryptography, quantum teleportation, dense coding, error correction and quantum computation [1]. A fundamental problem in quantum information theory is how to quantify the degree of entanglement in a practical and operational way 2,33 . One of the most meaningful and physically motivated measures is the entanglement of formation (EOF) 2, 3], which quantifies the minimal cost needed to prepare a certain quantum state in terms of EPR pairs. Related to the EOF the behavior of entanglement has recently been shown to play important roles in quantum phase transition for various interacting quantum many-body systems [4] and may significantly affect macroscopic properties of solids [5]. Moreover, it has been shown that there is a remarkable connection between entanglement and the capacity of quantum channels 6]. A quantitative evaluation of EOF is thus of great significance both theoretically and experimentally.

Considerable efforts have been spent on deriving EOF or its lower bound through analytical and numerical approaches, for some limited sets of mixed states 7, 8, 9, 10, 11, 12, 13, 14, 15, 16, 17. Among them, the most noteworthy results are an elegant analytical formula for two qubits [7, 8], explicit derivations for isotropic states 9], Werner states 11] and Gaussian states with certain symmetries [17]. Closed-form expressions have also been given for special classes of high dimensional states 12, 13, 14] and rotationally symmetric states in $2 \otimes n$ systems [15], where $n$ is the dimension of the Hilbert space associated with the second subsystem. Possible lower bounds have been given in 16] only for $2 \otimes n$ states. Notable progress has been achieved in [18, 19] in giving analytic lower bounds [that can be optimized further numerically [18]] for the concurrence, which permits to furnish a lower bound of EOF for a generic mixed state. However, this lower bound is not explicit except for the case of $2 \otimes n$ systems 19]. For low dimensional systems, numerical methods [10] can be used to estimate EOF. Nevertheless, they are generally time-consuming and often not very efficient. The notorious difficulty of evaluation for EOF is due to the complexity to solve a high dimensional optimization problem, which becomes a formidable task, as the dimensionality of the Hilbert space grows.

In this Letter, we present the first analytical calculation of a tight lower bound of EOF for arbitrary bipartite quantum states. An explicit expression for the bound is obtained from the convex hull of a simple function, based on a known result in [9]. This is achieved by establishing a key connection between EOF and two strong separability criteria, the Peres-Horodecki criterion [20, 21] and the realignment criterion [22, 23]. The bound is shown to be exact for some special states such as isotropic states [9, 24] and permits to provide EOF estimations for many bound entangled states (BES). It provides a very simple computable way for getting information on the actual value of $\mathrm{EOF}$, and in particular, fills significantly the large gap between the nice result on the two qubits case [7, 8] and a few other existing results (mentioned above) for high dimensional mixed states.

Let us first recall some useful notations. A pure $m \otimes n$ $(m \leq n)$ quantum state $|\psi\rangle$ is a normalized vector in the tensor product $\mathcal{H}_{A} \otimes \mathcal{H}_{B}$ of two Hilbert spaces $\mathcal{H}_{A}, \mathcal{H}_{B}$ for systems $A, B$. The entanglement of formation is defined to be $E(|\psi\rangle)=S\left(\rho_{A}\right)$ where $\rho_{A} \equiv \operatorname{Tr}_{B}(|\psi\rangle\langle\psi|)$ is the reduced density matrix. Here $S\left(\rho_{A}\right)$ is the entropy

$$
S\left(\rho_{A}\right) \equiv-\sum_{i=1}^{m} \mu_{i} \log _{2} \mu_{i}=H(\vec{\mu}),
$$

where $\mu_{i}$ are the eigenvalues of $\rho_{A}$ and $\vec{\mu}$ is the Schmidt vector $\left(\mu_{1}, \mu_{2}, \ldots, \mu_{m}\right)$. It is evident that $E(|\psi\rangle)$ vanishes only for product states. This definition can be extended to mixed states $\rho$ by the convex roof,

$$
E(\rho) \equiv \min _{\left\{p_{i},\left|\psi_{i}\right\rangle\right\}} \sum_{i} p_{i} E\left(\left|\psi_{i}\right\rangle\right)
$$

for all possible ensemble realizations $\rho=\sum_{i} p_{i}\left|\psi_{i}\right\rangle\left\langle\psi_{i}\right|$, where $p_{i} \geq 0$ and $\sum_{i} p_{i}=1$. Consequently, a state $\rho$ is separable if and only if $E(\rho)=0$ and hence can be represented as a convex combination of product states as $\rho=\sum_{i} p_{i} \rho_{i}^{A} \otimes \rho_{i}^{B}$, where $\rho_{i}^{A}$ and $\rho_{i}^{B}$ are pure state 
density matrices associated to the subsystems $A$ and $B$, respectively [25]. The measure Eq. (2) satisfies all the essential requirements of a good entanglement measure: convexity, no increase under local quantum operations and classical communications on average, no increase under local measurements, asymptotic continuity and other properties [2, 3].

A central idea for our approach is as follows: instead of the conventional method to make optimization subject to a large number of constraints in Eq. (2), we look for minimal admissible $E\left(\left|\psi_{i}\right\rangle\right)$ for a given $\left|\psi_{i}\right\rangle$ with some restrictions generated from the simple computable PeresHorodecki criterion of positivity under partial transpose (PPT criterion) 20, 21] and the realignment criterion 22, 23. One then expects to obtain a tight lower bound through a convex roof construction for the pure states. Without loss of generality, we suppose that one has a general pure $m \otimes n$ quantum state, which can always be written in the standard Schmidt form

$$
|\psi\rangle=\sum_{i} \sqrt{\mu_{i}}\left|a_{i} b_{i}\right\rangle
$$

where $\sqrt{\mu_{i}}(i=1, \ldots m)$ are the Schmidt coefficients, $\left|a_{i}\right\rangle$ and $\left|b_{i}\right\rangle$ are the orthonormal basis in $\mathcal{H}_{A}$ and $\mathcal{H}_{B}$, respectively. It can be straightforwardly verified that the reduced density matrices $\rho_{A}$ and $\rho_{B}$ have the same eigenvalues $\mu_{i}$. It follows from Eq. (11) that $E(|\psi\rangle)$ vanishes only for a product state and reaches its maximum $\log _{2} m$ for a maximally entangled state.

Let us recollect some details on the two above mentioned criteria. Peres made firstly a distinguished progress in the study of separability 20 by showing that $\rho^{T_{A}} \geq 0$ should be satisfied for a separable state, where $\rho^{T_{A}}$ stands for a partial transpose of $\rho$ with respect to the subsystem $A$. It was further shown by Horodecki et al. 21] that $\rho^{T_{A}} \geq 0$ is also sufficient for separability of $2 \otimes 2$ and $2 \otimes 3$ bipartite systems. In addition $\left\|\rho^{T_{A}}\right\|$ was shown in Refs. [20, 26] to be invariant under local unitary transformation (LU), where $\|\cdot\|$ stands for the trace norm defined by $\|G\|=\operatorname{Tr}\left(G G^{\dagger}\right)^{1 / 2}$. It is clear that $\rho=\sum_{i j k l} \rho_{i k, j l}\left|a_{i} b_{k}\right\rangle\left\langle a_{j} b_{l}\right|$ and $\rho_{i k, j l}^{T_{A}}=\rho_{j k, i l}$ in a suitably chosen orthonormal basis $a_{i}(i \leq m)$ and $b_{k}$ $(k \leq n)$. Here the subscripts $i$ and $j$ can be regarded as the row and column indices for the subsystem $A$ respectively, while $k$ and $l$ are such indices for the subsystem $B$.

The realignment criterion is another powerful operational criterion for separability given in 22, 23]. It demonstrates a remarkable ability to detect many BES [22, 23] and even genuinely tripartite entanglement 27]. Recently considerable efforts have been made in proposing stronger variants and multipartite generalizations for this criterion 28]. The criterion says that a realigned version $\mathcal{R}(\rho)$ of $\rho$ should satisfy $\|\mathcal{R}(\rho)\| \leq 1$ for any separable state $\rho$. $\mathcal{R}(\rho)$ is simply defined to be $\mathcal{R}(\rho)_{i j, k l}=\rho_{i k, j l}$ [22, 23, 27]. The LU invariant property holds also for
$\|\mathcal{R}(\rho)\|$ 23]. For the pure state of Eq. (3), it is straightforward to prove

$$
\left\|\rho^{T_{A}}\right\|=\|\mathcal{R}(\rho)\|=\left(\sum_{i} \sqrt{\mu_{i}}\right)^{2}=\lambda,
$$

as already shown in 22, 26] and [19], where $\lambda$ varies from 1 to $m$.

Let us also review some important results in [9] which we will use for the proof of our main Theorem. Terhal and Vollbrecht gave the first formula for the entanglement of formation for a class of mixed states in arbitrary dimension $d$ : the isotropic states

$$
\rho_{F}=\frac{1-F}{d^{2}-1}\left(I-\left|\Psi^{+}\right\rangle\left\langle\Psi^{+}\right|\right)+F\left|\Psi^{+}\right\rangle\left\langle\Psi^{+}\right|,
$$

where $\left|\Psi^{+}\right\rangle \equiv \sqrt{1 / d} \sum_{i=1}^{d}|i i\rangle$ and $F=\left\langle\Psi^{+}\left|\rho_{F}\right| \Psi^{+}\right\rangle$, satisfying $0 \leq F \leq 1$, is the fidelity of $\rho_{F}$ and $\left|\Psi^{+}\right\rangle$. They found that for $F \geq 1 / d$, the EOF for isotropic states is $E\left(\rho_{F}\right)=\operatorname{co}[R(F)]$ where $R(F)$ is a simple function of $F$. Here the symbol "co" means the convex hull, which is the largest convex function that is bounded above by a given function. They have also presented an explicit expression of $\operatorname{co}[R(F)]$ for $d=2,3$, and conjectured its general form for arbitrary $d$.

With the above analysis and preparation, we can formulate the main result of this Letter:

Theorem. - For any $m \otimes n(m \leq n)$ mixed quantum state $\rho$, the entanglement of formation $E(\rho)$ satisfies

$$
E(\rho) \geq \operatorname{co}[R(\Lambda)]
$$

where

$$
\begin{aligned}
& R(\Lambda)=H_{2}[\gamma(\Lambda)]+[1-\gamma(\Lambda)] \log _{2}(m-1), \\
& \gamma(\Lambda)=\frac{1}{m^{2}}[\sqrt{\Lambda}+\sqrt{(m-1)(m-\Lambda)}]^{2},
\end{aligned}
$$

with $\Lambda=\max \left(\left\|\rho^{T_{A}}\right\|,\|\mathcal{R}(\rho)\|\right)$ and $H_{2}($.$) is the standard$ binary entropy function.

Proof. - To obtain the desired lower bound, we assume that one has already found an optimal decomposition $\sum_{i} p_{i} \rho^{i}$ for $\rho$ to achieve the infimum of $E(\rho)$, where $\rho^{i}$ are pure state density matrices. Then $E(\rho)=\sum_{i} p_{i} E\left(\rho^{i}\right)$ by definition. For a pure state density matrix $\sigma=|\psi\rangle\langle\psi|$ with $|\psi\rangle$ given by Eq. (3), one has $\left\|\sigma^{T_{A}}\right\|=\|\mathcal{R}(\sigma)\|=$ $\left(\sum_{k=1}^{m} \sqrt{\mu_{k}}\right)^{2}=\lambda$ according to Eq. (4). We would like firstly to find a minimal admissible $H(\vec{\mu})$ for a given $\lambda$. This minimization problem has been solved in [9],

$$
\begin{aligned}
R(\lambda) & =\min _{\vec{\mu}}\left\{H(\vec{\mu}) \mid\left(\sum_{k=1}^{m} \sqrt{\mu_{k}}\right)^{2}=\lambda\right\} \\
& =H_{2}[\gamma(\lambda)]+[1-\gamma(\lambda)] \log _{2}(m-1),
\end{aligned}
$$

where

$$
\gamma(\lambda)=\frac{1}{m^{2}}[\sqrt{\lambda}+\sqrt{(m-1)(m-\lambda)}]^{2} .
$$


The function $R(\lambda)$ here is the $R(F)$ used in [9] after substitutions of $d \rightarrow m, F \rightarrow \lambda / m$. It is further shown in [9] that $\operatorname{co}[R(\lambda)]$ is a monotonously increasing, convex function and satisfies $\operatorname{co}[R(\lambda)] \leq H(\vec{\mu})$ for a given $\lambda$. Denote $\mathcal{E}(\lambda)=\operatorname{co}[R(\lambda)]$, one thus has

$$
\begin{aligned}
E(\rho) & =\sum_{i} p_{i} E\left(\rho^{i}\right)=\sum_{i} p_{i} H\left(\vec{\mu}^{i}\right) \geq \sum_{i} p_{i} \mathcal{E}\left(\lambda^{i}\right) \\
& \geq \mathcal{E}\left(\sum_{i} p_{i} \lambda^{i}\right) \geq\left\{\begin{array}{l}
\mathcal{E}\left(\left\|\rho^{T_{A}}\right\|\right), \\
\mathcal{E}(\|\mathcal{R}(\rho)\|),
\end{array}\right.
\end{aligned}
$$

where we have used the monotonicity and convexity properties of $\mathcal{E}$, and convexity of the trace norm $\left\|\rho^{T_{A}}\right\| \leq$ $\sum_{i} p_{i}\left\|\left(\rho^{i}\right)^{T_{A}}\right\|$ and $\|\mathcal{R}(\rho)\| \leq \sum_{i} p_{i}\left\|\mathcal{R}\left(\rho^{i}\right)\right\|$. Set $\Lambda=$ $\max \left[\left\|\rho^{T_{A}}\right\|,\|\mathcal{R}(\rho)\|\right]$, we arrive at

$$
E(\rho) \geq \mathcal{E}(\Lambda)=\operatorname{co}[R(\Lambda)] .
$$

which gives exactly the conclusion of Eq. (6).

Since $\operatorname{co}[R(\lambda)]$ is the largest convex function that is nowhere larger than $R(\lambda)$, it is optimal to give the best lower bound according to the relations Eqs. (8), (10), and (11). From the general form for $\operatorname{co}[R(\lambda)]$ given in $[9]$, the following relation

$$
\begin{aligned}
& E(\rho) \geq \\
& \begin{cases}0, & \Lambda=1, \\
H_{2}[\gamma(\Lambda)]+[1-\gamma(\Lambda)] \log _{2}(m-1), & \Lambda \in\left[1, \frac{4(m-1)}{m}\right], \\
\frac{\log _{2}(m-1)}{m-2}(\Lambda-m)+\log _{2} m, & \Lambda \in\left[\frac{4(m-1)}{m}, m\right],\end{cases}
\end{aligned}
$$

holds for $m=2,3$. We have strong evidence for its correctness for arbitrary $m$ by verifying directly, that the second derivative for $R(\lambda)$ with respect to $\lambda$ goes from positive to negative value with only one zero point when $\lambda$ varies from 1 to $m$ according to the analysis of $[9]$.

The result of the above Theorem and its general expression Eq. (12) provide an explicit tight lower bound for the EOF without the need of any numerical optimization procedure. In fact, it can be done in an entirely straightforward manner through the computation of the trace norm of a certain matrix by standard linear algebra packages. Some further significant features arising from our general result are illustrated in several examples and the following discussions.

Example 1: Qubit-qudit system

When $m=2$, which corresponds to a qubit-qudit system, one derives easily from the Theorem and Eq. (12) that

$$
E(\rho) \geq \begin{cases}0, & \Lambda=1 \\ H_{2}\left[\frac{1}{2}\left(1+\sqrt{1-(\Lambda-1)^{2}}\right)\right], & \Lambda \in[1,2]\end{cases}
$$

This recovers previous results obtained by the authors in 19], where $\Lambda=\max \left[\left\|\rho^{T_{A}}\right\|,\|R(\rho)\|\right]$, and others in [29], where $\Lambda=\left\|\rho^{T_{A}}\right\|$. In addition Eq. (13) can detect and give lower bounds of EOF for all entangled states of two qubits and of qubit-qutrit systems, since the PeresHorodecki criterion is necessary and sufficient for separability in these cases 21]. Furthermore, whenever there is a two qubit state in which $\Lambda-1$ is equal to the concurrence defined in [8], the bound Eq. (13) will give the exact value of EOF. For example the $2 \otimes 2$ Werner state 11] fits this condition by direct verification. The bound Eq. (13) will be particularly useful for the study of entanglement in realistic many-body physical systems. For example, one usually needs to monitor entanglement dynamics and distribution between a spin $1 / 2$ particle and the remaining parts for a solid state system or a quantum computing device, and our bound can be useful in this context.

\section{Example 2: Isotropic states}

Isotropic states Eq. (5) were firstly proposed in 24] and further properties were analyzed in 11]. They arise naturally in some special depolarizing channel 24] and constitute the class of $U \otimes U^{*}$ invariant mixed states in $d \otimes d$ systems. These states have been shown to be separable for $F \leq 1 / d$ 24]. The EOF $E(\rho)$ for this class of states has been given in 9 by an elegant extremization procedure. It is derived in 22, 26] that $\left\|\rho_{F}^{T_{A}}\right\|=\left\|\mathcal{R}\left(\rho_{F}\right)\right\|=d F$ for $F>1 / d$. Thus one can directly exploit the above Theorem with $\Lambda=d F$ to see that the bound given in Eqs. (6) and (12) coincides with the exact value of EOF for the whole class of states in Eq. (5).

Our Theorem and the general relation of Eq. (12) complement a number of existing approaches to make a quite good estimate of entanglement for BES, benefiting from the powerful realignment criterion which enables one to detect many of the BES [22, 23].

The bound can be made even better if it is much easier to compute a convex-roof extended entanglement measure [22, 30] $\left\|\rho^{T_{A}}\right\|_{\text {co }}$ or $\|\mathcal{R}(\rho)\|_{\text {co }}$, than the EOF. The extended measures in our case are defined by $\left\|\rho^{T_{A}}\right\|_{\text {co }} \equiv \min _{\left\{p_{i}, \rho^{i}\right\}} \sum_{i} p_{i}\left\|\left(\rho^{i}\right)^{T_{A}}\right\|$ and $\|\mathcal{R}(\rho)\|_{\text {co }} \equiv$ $\min _{\left\{p_{i}, \rho^{i}\right\}} \sum_{i} p_{i}\left\|\mathcal{R}\left(\rho^{i}\right)\right\|$ where $\rho=\sum_{i} p_{i} \rho^{i}, p_{i} \geq 0$ and $\sum_{i} p_{i}=1$. They have been studied and calculated for some special class of states in 22, 30]. Defining $\Lambda=\max \left(\left\|\rho^{T_{A}}\right\|_{\text {co }},\|\mathcal{R}(\rho)\|_{\text {co }}\right)$, one finds that the result of the Theorem is still valid, since the last inequality in Eq. (10) holds as $\mathcal{E}\left(\sum_{i} p_{i} \lambda^{i}\right) \geq \mathcal{E}(\Lambda)$. This will provide a tighter lower bound for the EOF since generally $\left\|\rho^{T_{A}}\right\|_{\text {co }} \geq\left\|\rho^{T_{A}}\right\|$ and $\|\mathcal{R}(\rho)\|_{\text {co }} \geq\|\mathcal{R}(\rho)\|$ follow from the definitions.

Due to the nonanalytic behavior of the right hand side of Eq. (12), it is difficult to find a specific condition under which our bound will be exact for a general state. Roughly, for a $2 \otimes n$ system one necessary requirement is that all the $\rho^{i}$ should be equally entangled with $E\left(\rho^{i}\right)=R(\Lambda)$ in an optimal decomposition for achieving $\mathrm{EOF}$, as in this case we demand all the inequalities in Eq. (10) to be changed into equalities. In higher dimen- 
sions, it is necessary that all the $\rho^{i}$ should have equal EOF of $R(\Lambda)$ when $1 \leq \Lambda \leq 4(m-1) / m$ while there should be two values of EOF, $\log _{2} m$ and $R(4(m-1) / m)$ for $4(m-1) / m \leq \Lambda \leq m$, as seen from Eq. (12).

Although the work 19] permits to furnish a lower bound for EOF (in fact there is no explicit formula given there), it is by no means optimal for general states except for $2 \otimes n$ systems. Instead, the procedure in 19] clearly imposes more restrictions than that of the present work: it requires firstly to give a lower bound of concurrence for a given $\Lambda$, and then obtain a possible lower bound of EOF from the derived concurrence bound. Thus the result in the present Letter is optimal as long as the parameter $\Lambda$ is involved only, since we have utilized the largest convex function $\operatorname{co}[R(\lambda)]$ that is bounded above by $R(\lambda)$.

In summary, we have determined a completely analytic lower bound of EOF for an arbitrary bipartite mixed state, which characterizes optimally the quantitative behavior of entanglement through the well-known PeresHorodecki criterion and the realignment criterion for separability. The procedure only involves a simple computation of matrix eigenvalues and can be done efficiently with the standard linear algebra packages. Our bound leads to exact values of EOF for some special quantum states and enables one to give an easy EOF evaluation for many BES, a task which was extremely difficult before. We are of the opinion that the result constitutes a significant bridge over the big gap between the elegant result of Wootters for 2 qubits, and the few existing results mentioned before for high dimensional states. In this way our method can yield a powerful tool for investigating quantitatively the character of entanglement for practical laboratory sources (atomic, photonic, spin or other carriers), and for the study of many-body systems. Furthermore, it may provide important insights into realistic quantum channels and condensed matter systems, revealing deep connections between entanglement and macroscopic properties for the corresponding physical systems.

K.C. gratefully acknowledges support from the Alexander von Humboldt Foundation. The support of this work by the Deutsche Forschungsgemeinschaft SFB611 and German(DFG)-Chinese(NSFC) Exchange Programme 446CHV113/231 is also gratefully acknowledged. Partially supported by NKBRPC (2004CB318000).

[1] M.A. Nielsen and I.L. Chuang, Quantum Computation and Quantum Information (Cambridge University Press, Cambridge, England, 2000).

[2] C.H. Bennett, D.P. DiVincenzo, J.A. Smolin, and W.K. Wootters, Phys. Rev. A 54, 3824 (1996).
[3] M. Horodecki, Quant. Inf. Comp. 1, 3 (2001); D. Bruß, J. Math. Phys. 43, 4237 (2002); M.B. Plenio and S. Virmani, quant-ph/0504163

[4] A. Osterloh et al., Nature 416, 608 (2002); L.-A. Wu, M.S. Sarandy, and D.A. Lidar, Phys. Rev. Lett. 93, 250404 (2004).

[5] S. Ghosh, T.F. Rosenbaum, G. Aeppli, S.N. Coppersmith, Nature 425, 48 (2003); V. Vedral, Nature 425, 28 (2003).

[6] P.W. Shor, Commun. Math. Phys. 246, 453 (2004); A.A. Pomeransky, Phys. Rev. A 68, 032317 (2003).

[7] S. Hill and W.K. Wootters, Phys. Rev. Lett. 78, 5022 (1997).

[8] W.K. Wootters, Phys. Rev. Lett. 80, 2245 (1998).

[9] B.M. Terhal and K.G.H. Vollbrecht, Phys. Rev. Lett. 85, 2625 (2000).

[10] K. Audenaert, F. Verstraete, and B. De Moor, Phys. Rev. A 64, 052304 (2001); J.R. Gittings, A.J. Fisher, quant-ph/0302018

[11] K.G.H. Vollbrecht and R.F. Werner, Phys. Rev. A 64, 062307 (2001).

[12] G. Vidal, W. Dür, and J.I. Cirac, Phys. Rev. Lett. 89, 027901 (2002).

[13] S.M. Fei, J. Jost, X.Q. Li-Jost, and G.F. Wang, Phys. Lett. A 310, 333 (2003).

[14] S.M. Fei and X.Q. Li-Jost, Rep. Math. Phys. 53, 195 (2004).

[15] K.K. Manne and C.M. Caves, quant-ph/0506151

[16] P.X. Chen et al., Phys. Lett. A 295, 175 (2002); E. Gerjuoy, Phys. Rev. A 67, 052308 (2003); A. Łoziński et al., Europhys. Lett. 62, 168 (2003).

[17] G. Giedke, M.M. Wolf, O. Krüger, R.F. Werner, and J.I. Cirac, Phys. Rev. Lett. 91, 107901 (2003).

[18] F. Mintert, M. Kuś, and A. Buchleitner, Phys. Rev. Lett. 92, 167902 (2004).

[19] K. Chen, S. Albeverio, and S.M. Fei, Phys. Rev. Lett. 95, 040504 (2005).

[20] A. Peres, Phys. Rev. Lett. 77, 1413 (1996).

[21] M. Horodecki, P. Horodecki, and R. Horodecki, Phys. Lett. A 223, 1 (1996).

[22] O. Rudolph, quant-ph/0202121

[23] K. Chen and L.A. Wu, Quant. Inf. Comp. 3, 193 (2003).

[24] M. Horodecki and P. Horodecki, Phys. Rev. A 59, 4206 (1999).

[25] R.F. Werner, Phys. Rev. A 40, 4277 (1989).

[26] G. Vidal and R.F. Werner, Phys. Rev. A 65, 032314 (2002).

[27] M. Horodecki, P. Horodecki, and R. Horodecki, quant-ph/0206008

[28] K. Chen and L.A. Wu, Phys. Lett. A 306, 14 (2002); O. Rudolph, Physical Review A 67, 032312 (2003); S. Albeverio, K. Chen, and S.M. Fei, Phys. Rev. A 68, 062313 (2003); K. Chen and L.A. Wu, Phys. Rev. A 69, 022312 (2004); H. Fan, quant-ph/0210168 P. Wocjan and M. Horodecki, quant-ph/0503129 L. Clarisse, P. Wocjan, quant-ph/0504160

[29] D.P. Chi and S. Lee, J. Phys. A 36, 11503 (2003).

[30] S. Lee, D.P. Chi, S.D. Oh, and J. Kim, Phys. Rev. A. 68, 062304 (2003). 